\title{
A new dewatering technique for stingless bees honey
}

\author{
Ahmad Syazwan Ramli ${ }^{1}$, Firdaus Basrawi ${ }^{1, *}$, Daing Mohamad Nafiz Daing Idris ${ }^{1}$, Mohd \\ Hazwan bin Yusof ${ }^{1}$, Thamir Khalil Ibrahim ${ }^{1}$, Zulkifli Mustafa ${ }^{2}$, and Shaharin A. Sulaiman ${ }^{3}$ \\ ${ }^{1}$ Meliponini Engineering Laboratory (MepEL), Energy Sustainability Focus Group (ESFG), Faculty \\ of Mechanical Engineering, Universiti Malaysia Pahang \\ ${ }^{2}$ Universiti Sains Malaysia, Kubang Kerian, Kelantan, Malaysia \\ 3Department of Mechanical Engineering, Universiti Teknologi Petronas, Bandar Seri Iskandar, \\ 31750, Tronoh, Perak, Malaysia
}

\begin{abstract}
One of the problems faced in stingless bee honey storage is spoilage by the fermentation process occurs in honey due to its high water content. There are a few techniques available currently, but they are time consuming and there is excessive heat involved in the process. The temperature of the process must be kept low because excessive heat can deteriorate nutrition value and biochemical content in honey. Hence, a new method of honey dewatering was developed using a Low Temperature Vacuum Drying (LTVD) with induced nucleation technique. The objective of this research is to investigate the performance of a LTVD with induced nucleation to reduce the water content in honey. First, the honey was placed in a pressure vessel, and then air was removed. Then, the honey was slightly heated at $30^{\circ} \mathrm{C}$ and the water content before and after the experiment was measured by a refractometer. The steps were repeated until the water content reached below $20 \%$. It was found that the LTVD method improved the water removal rate significantly with an average of $0.15 \%$ of water content per minute. That is 3 times much faster than the conventional method of low temperature heating by Tabouret. Higher temperature during dewatering process improved the dewatering rate significantly. It can be concluded that LTVD is a promising option in tackling the high water content in stingless bee honey issue.
\end{abstract}

\section{Introduction}

Honey is a very sweet flavour natural liquid made by bees from nectar which is taken from flowers or from secretions of living parts from plants [1]. It can consist up to $65 \%$ sugar which is primarily fructose and glucose [2]. The taste and aroma of the honey are ranging in color from pale yellow to dark amber which varies according to geographical and seasonal conditions [3]. In addition, the water content of honey in humid area is normally much higher than the low humid area [4]. According to Marinus (2006), honey from stingless bee in Trinidad for example has a very high water content which can reach up to $42 \%$ [5]. The water content in honey especially in stingless bee honey in general has become a major issue since the high water content promotes microbial activities that causes fermentation process to occur 
in honey as reported by Stephen (1946)[6]. On the other hand, honey is considered to be a heat sensitive material in general with most of its content can altered due to certain amount of excessive heat especially the antimicrobial antioxidant in it as explained by Fauzi et al. 2014[7]. Turhan et al. (2008) stated that excessive heating may also cause crystallization which will decrease the quality of the honey as well [8]. It was also found by Kretavičius et al. (2010) that heating the honey lower than $50^{\circ} \mathrm{C}$ will not damage the enzyme activity in the honey [9].

Therefore, in recent years there has been a lot of effort to implement low temperature drying for heat sensitive material in order to preserve the quality, nutrients and other qualities that might have change or damage by high temperature heating. In order to lower the temperature for water removing purpose, the process is subjected to low pressure or vacuum condition so that the boiling point of water can be lowered down as low as possible. Hence this combination is commonly known as low temperature vacuum drying (LTVD). In general, vacuum drying always involves with low temperature since by introducing vacuum condition, the boiling point of water is definitely lower than $100^{\circ} \mathrm{C}$. Hence the term low temperature for vacuum drying is often referring that only a little heat is supplied so that the temperature for the drying process is lower than $100^{\circ} \mathrm{C}$. LTVD is a common method used to dehydrate and remove the water content from heat sensitive and high quality mediums such as food biomedical and pharmaceuticals products for preservation purpose before they reach consumers in the market. Since only low temperature is used in the drying process, there is no excessive heat that could damage the heat sensitive material such as stingless bee honey in this case.

But there are currently many variants of LTVD available. For instance, there is also superheated steam drying developed by Devahastin et al. (2004) which also utilizes the LTVD concept. However, this method takes too much time for dewatering viscous liquid such as honey [10]. There is also freeze drying LTVD which the medium is first frozen before it is slightly heated to sublimate the water content but the freezing process consumes high amount of energy and not very efficient as stated by Nam et. al. (2007)[11]. There is also spray drying combined with LTVD but this method can only convert the liquid medium directly to solid and the dewatering level cannot be controlled [12]. There was also an attempt to assist LTVD with microwave to enhance the vaporization of water. However, this method will damage the composition and nutrient of the medium due the high radiation energy emitted from the microwave [13]. It is proposed that LTVD should be combined with nucleation boiling to enhance the rate of honey dewatering. A LTVD with induced nucleation boiling has been developed to meet this requirement of maintaining the original content of nutrients, quality, aroma and taste of the honey throughout the dewatering process. From literature reviewed, there is no study reported on the LTVD with nucleation boiling especially on the effect of temperature on dewatering process. Thus the objective of this research is to investigate the performance of a LTVD with induced nucleation to reduce the water content in honey.

\section{Materials and Method}

For the first phase of this research, an experimental test rig was prepared to properly measure the effectiveness of the honey dewatering process. Fig. 1 depicts the schematic diagram of the experimental setup. 


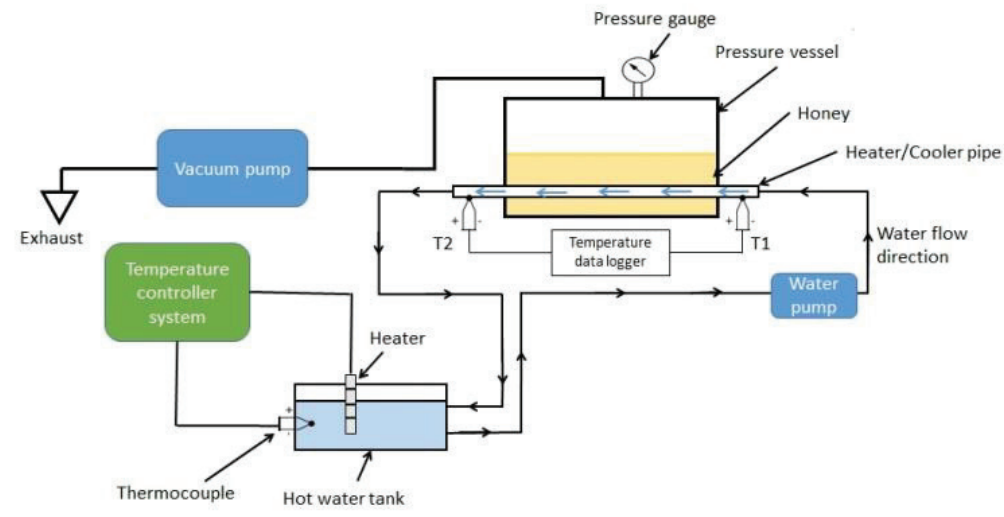

Fig. 1. Schematic diagram of the experimental setup

The main part of this setup was the pressure vessel where it the sample for LTVD is placed. A vacuum pump was connected to the vessel which created a vacuum condition inside the vessel. For this experiment, a cylindrical container type of vessel was chosen. A pressure gauge attached to the vessel is used to monitor the pressure inside the vessel. The right vacuum pressure and temperature are crucial to induce the nucleation in honey. There was also a stainless steel pipe inside the vessel which acts as a heating element for the sample. A thermocouple was attached at the water inlet and another one at the outlet of the stainless steel pipe. Both of these thermocouples are attached to a temperature data logger where the water inlet temperature, $T_{1}$ and the water outlet temperature, $T_{2}$ were used to monitor the heat transfer from the hot water to the honey.

For heating up the sample, a heater in a water tank heated up the water up to the determined temperature and the water temperature of the tank was held constant by a temperature controlling system with a thermocouple. For this experiment, $30^{\circ} \mathrm{C}$ was set for the dewatering. The honey samples used in this experiment was obtained from a farm in the state of Perak, Malaysia. Fig. 2 shows the sample obtained from a farm in Perak. The water content of the honey sample was measured by a refractometer. Fig. 3 is an example of measurement taken by a refractometer. The initial reading for the sample was around $26 \%$ of water content.

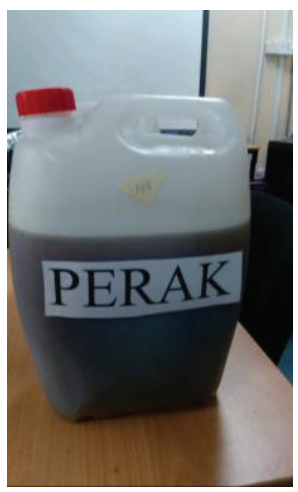

Fig. 2. Stingless bee honey samples from Perak in liquid container. 

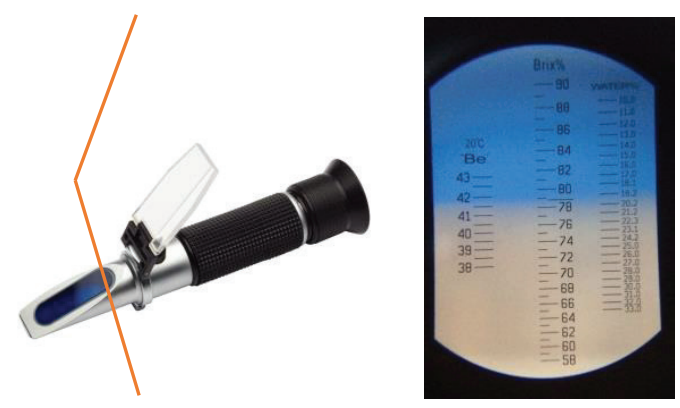

Fig. 3. Measurement sample by a refractometer.

The experiment was done by introducing the honey sample inside the pressure vessel and closes the lid tightly. 200g of the honey sample was weighted first as shown in Fig. 4. Then the reading of the initial water content of the sample was taken by using a refractometer. After 10 minutes, the experiment was stopped to measure the final water content. The process was repeated until the water content of the sample reaches $20 \%$ level or below.

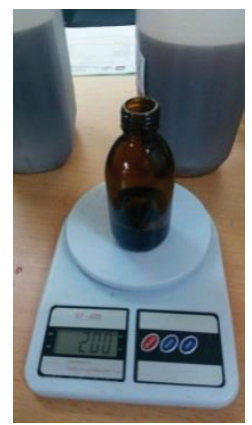

Fig. 4. Honey sample was put in a bottle and weighted for $200 \mathrm{~g}$.

The data that needs to be collected for this research is the water content of the sample against 10 minutes time interval. In this case, the temperature of heater, heat transfer rate in honey and the pressure inside the vessel were assumed to be constant. By having the water content data against time, the rate of water removal can be determined for each parameter by using equation (1) below:

$$
\eta_{D}=\frac{\Delta W_{h}}{t}
$$

where $\eta_{D}$ is the dewatering [ $\left.\% / \mathrm{min}\right], \Delta W_{h}$ is the change of water content [\%], and $t$ is the time taken for the dewatering process [min].

\section{Results and Discussion}

During the experiment, nucleation bubbling was observed in the honey sample. The nucleation occurred in the sample was observed and the view of the nucleation density is recorded via a camera. Fig. 5 is an example of the nucleation bubbling that occurred during the dewatering. For this experiment, the size, density and shape of the nucleation was considered to be constant. 


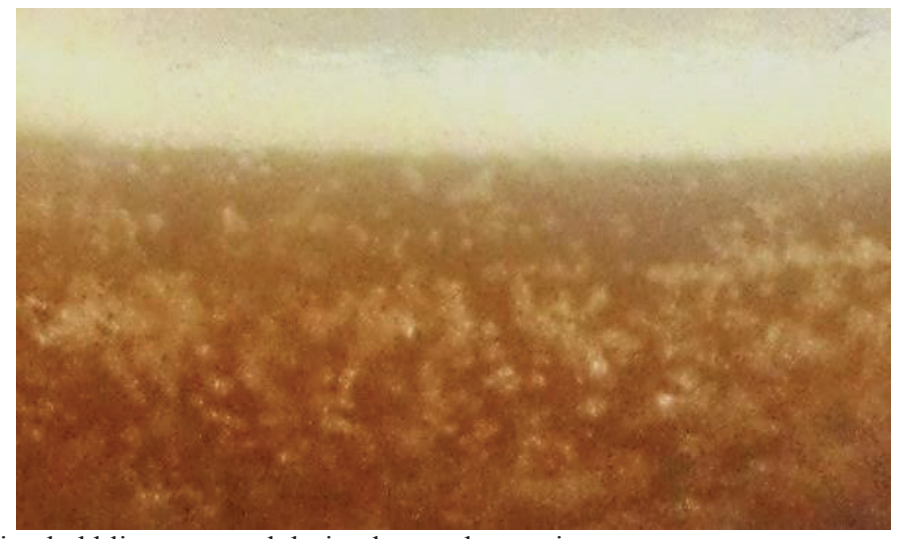

Fig. 5. Nucleation bubbling occurred during honey dewatering.

Fig. 6 shows water content changes by time for each experiment. As shown in Fig. 6, the increase of temperature improved the rate of dewatering significantly. At temperature of $30^{\circ} \mathrm{C}$, the dewatering reached the desired level of water content within 60 minutes. The average dewatering rate, $\eta_{D}$ with the temperature of $30^{\circ} \mathrm{C}$ was $0.1 \% / \mathrm{min}$.

If the dewatering rate is compared to the traditional method of using hot air stream for example, this dewatering method is much better because the hot air stream method takes about 2 hour to reduce approximately $2 \%$ of water content. So the dewatering rate is about $\eta_{D}=0.017$. On top of that, the hot air is very difficult to control and may damage the content of the honey [14]. A large dehumidifier built by Paysen for example can dewater 57 tonnes of honey but the dewatering rate is only $0.6 \%$ of water content within 30 hour [15].The dewatering rate for this method is approximately $\eta_{D}=0.00033$. With the help of low pressure vacuum, Tabouret managed to remove the water content at the rate of $\eta_{D}=0.048 \%$ per minute but at a temperature of $63^{\circ} \mathrm{C}$ which is considered to be too high for dewatering process[16].This means that induced nucleation LTVD performed 3 times much faster than the result achieved by Tabouret. By using LTVD with the assistance of microwave on the other hand, the dewatering rate is much higher which is around $\eta_{D}=0.25 \%$ per minute but the radiation emitted by the microwave have the potential to damaged and alter the original quality, aroma and taste of the honey. Fig. 7 compares dewatering rate of present result with other techniques

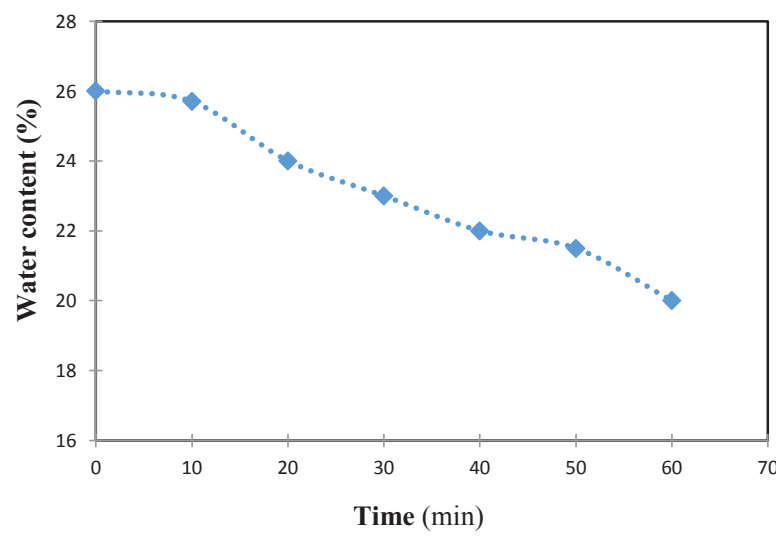

Fig. 6. Dewatering result for honey sample at $30^{\circ} \mathrm{C}$ operating temperatures. 


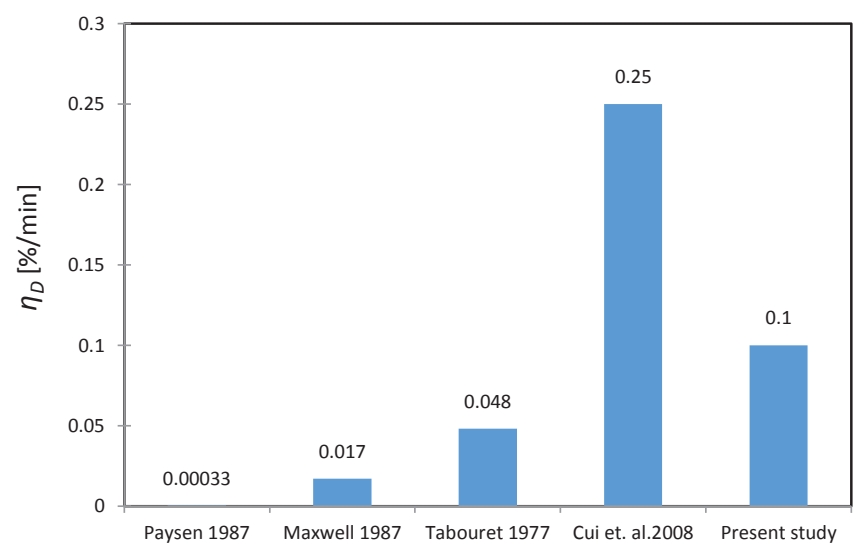

Fig. 7. Comparison of the experiment's dewatering rate with other techniques.

\section{Conclusion}

The effectiveness of LTVD with induced nucleation at $30^{\circ} \mathrm{C}$ for dewatering stingless bee honey was investigated, and the performance was compared with other dewatering methods. It was found that by introducing the nucleation boiling in LTVD, the performance and efficiency of the dewatering rate can be further improved for up to an average of 3 times faster than the traditional methods without the need to increase any energy and operational cost. The $\eta_{D}$ for induced nucleation LTVD was $\eta_{D}=0.1$ at temperature $30^{\circ} \mathrm{C}$. This means that the method is much better compared to dehumidifier method which only had $\eta_{D}=$ 0.00033 , hot air drying with $\eta_{D}=0.017$ and low pressure heating with $\eta_{D}=0.048$. The rate of microwave LTVD is much better which is $\eta_{D}=0.25$ but the radiation generated by the microwave damaged the nutritional content in honey. However, further testing to check the content of the honey before and after the proposed LTVD with induced nucleation should be done to verify that this method does not give any side effect or any damage to the original content of the honey.

The authors would like to thank the Ministry of Education Malaysia for providing Research Grant under FRGS. The authors gratefully acknowledge Prof. Ir. Dr. Hassan Ibrahim of the Faculty of Mechanical Engineering, University Malaysia Pahang for his kind advice and the help in editing this manuscript.

\section{References}

1. Mendes, E., Brojo Proença, E., Ferreira, I. M. P. L. V. ., and Ferreira, M. ., Quality evaluation of Portuguese honey, Carbohydr. Polym. 3 (1998) 219-223.

2. Nurhadi, B., and Roos, Y. H., Water Sorption and Water Plasticization Behavior of Vacuum Dried Honey, Int. J. Food Prop. 19 (2016) 1370-1380.

3. Aparna, A. R., and Rajalakshmi, D., Honey - its characteristics, sensory aspects, and applications, Food Rev. Int. 15 (1991) 455-471.

4. Chen, G., Sun, X., Huang, Y., and Chen, K., Tracking the dehydration process of raw honey by synchronous two-dimensional near infrared correlation spectroscopy, J. Mol. Struct. 1076 (2014) 42-48.

5. Marinus, J. S., Water content of stingless bee honeys (Apidae, Meliponini): interspecific variation and comparison with honey of Apis mellifera, Apidologie 37 
(2006) 480-486.

6. Stephen, W. A., The Relationship of Moisture Content and Yeast Count in Honey Fermentation 1, Sci. Agric. 6 (1946) 258-264.

7. Silva, F., Fauzi, N. A., and Farid, M. M., High-Pressure Processing of Manuka Honey- Improvement of Antioxidant Activity, Preservation of Colour and Flow Behaviour, Food Bioprocess Technol. 7 (2014) 2299-2307.

8. Turhan, I., Tetik, N., Karhan, M., Gurel, F., and Tavukcuoglu, H. R., Quality of honeys influenced by thermal treatment, LWT-Food Sci. Technol. 41 (2008) 13961399.

9. Kretavičius, J., Kurtinaitienè, B., Racys, J., and Ceksteryte, V., Inactivation of glucose oxidase during heat-treatment de-crystallization of honey, Žemdirbyste (Agriculture) 4 (2010) 115-122.

10. Devahastin, S., Suvarnakuta, P., Soponronnarit, S., and Mujumdar, A. S., A Comparative Study of Low-Pressure Superheated Steam and Vacuum Drying of a Heat-Sensitive Material, Dry. Technol. 22 (2004) 1845-1867.

11. Nam, J. H., and Song, C. S., Numerical simulation of conjugate heat and mass transfer during multi-dimensional freeze drying of slab-shaped food products, Int. J. Heat Mass Transf. 23 (2007) 4891-4900.

12. Cuevas-Glory, L. F. P., A., J., Sosa-Moguel, O., Sauri-Duch, E., and BringasLantigua, M., Optimization of the Spray-Drying Process for Developing Stingless Bee Honey Powder, Int. J. Food Eng. 13 (2017).

13. Cui, Z., Sun, L., Chen, W., and Sun, D., Preparation of dry honey by microwavevacuum drying, J. Food Eng. 84 (2008) 582-590.

14. Crane, E., The removal of water from honey, Bee World 77 (1996) 120-129.

15. Tabouret, T., Vacuum drying of honey, Apiacta 12 (1977) I57-164. 